\title{
Impact of strain-induced electronic topological transition on the thermoelectric properties of $\mathrm{PtCoO}_{2}$ and $\mathrm{PdCoO}_{2}$
}

\author{
Markus Ernst Gruner, ${ }^{1,2,{ }^{*}}$ Ulrich Eckern, ${ }^{3}$ and Rossitza Pentcheva ${ }^{2}$ \\ ${ }^{1}$ Forschungs-Neutronenquelle Heinz Maier-Leibnitz (FRM II), Technische Universität München, 85748 Garching, Germany \\ ${ }^{2}$ Faculty of Physics and Center for Nanointegration, CENIDE, University of Duisburg-Essen, 47048 Duisburg, Germany \\ ${ }^{3}$ Institute of Physics, University of Augsburg, 86135 Augsburg, Germany
}

(Received 26 September 2015; published 22 December 2015)

\begin{abstract}
By a combination of first-principles calculations and semiclassical Boltzmann transport theory, we investigate the effect of epitaxial strain on the electronic structure and transport properties of $\mathrm{PtCoO}_{2}$ and $\mathrm{PdCoO}_{2}$. In contrast to the rather uniform elastic response of both systems, we predict for $\mathrm{PtCoO}_{2}$ a high sensitivity of the out-of-plane transport properties to strain, which is not present in $\mathrm{PdCoO}_{2}$. At ambient temperature we identify a considerable absolute change in the thermopower from $-107 \mu \mathrm{V} / \mathrm{K}$ at $-5 \%$ compressive strain to $-303 \mu \mathrm{V} / \mathrm{K}$ at $+5 \%$ tensile strain. This remarkable response is related to distinct changes of the Fermi surface, which involve the crossing of two additional bands at a moderate compressive in-plane strain. Combining our transport results with available experimental data on electrical and lattice thermal conductivity we predict a thermoelectric figure of merit of up to $Z T=0.25$ at $T=600 \mathrm{~K}$ for strained $\mathrm{PtCoO}_{2}$.
\end{abstract}

DOI: 10.1103/PhysRevB.92.235140

PACS number(s): 71.18.+y, 71.20.Be, 72.15.Eb, 72.15.Jf

\section{INTRODUCTION}

Recently, the quest for new thermoelectric materials beyond $\mathrm{Bi}_{2} \mathrm{Te}_{3}$ has gained considerable momentum which is owed to the increasing need of highly performing materials for energy harvesting and conversion. The most promising material classes identified so far are found among the semiconductors, which allow a careful adjustment of charge carrier type and concentration in order to achieve the optimum balance between thermopower, electrical, and thermal conductivity [1,2]. Together with the idea to exploit low-dimensional quantum-well structures to increase the thermoelectric performance [3], this leads to a series of new high performance materials which are formed of heterostructures or quantum dot superlattices [4-6]. Oxides, such as $\mathrm{Na}_{x} \mathrm{CoO}_{2}$ type or $\mathrm{Ca}_{3} \mathrm{Co}_{4} \mathrm{O}_{9}$ [7-11], represent another promising material class with the advantage of nontoxicity and abundance of their components in combination with the chemical and thermal stability rather than record-breaking performance values.

Low dimensionality is readily realized in naturally layered structures, which are for instance found in a specific class of cobaltates, the delafossites. Among those hexagonal $\mathrm{ABO}_{2}$ compounds, $\mathrm{PdCoO}_{2}$ and $\mathrm{PtCoO}_{2}$ take a special position, since these are-unlike most of the oxides, which are semiconducting or insulating-very good metallic conductors [12-14]. Such a material usually disqualifies for thermoelectric applications [1], but it was discovered early that the conductivity of $\mathrm{PdCoO}_{2}$ and $\mathrm{PtCoO}_{2}$ is highly anisotropic [12,15]. Recent theoretical work $[16,17]$ reports also a qualitative difference in the thermoelectric properties with respect to transport within the $a-b$ plane and along the hexagonal $c$ axis. $A b$ initio calculations predict significant negative values of about $-100 \mu \mathrm{V} \mathrm{K}^{-1}\left(\mathrm{PdCoO}_{2}\right)$ and $-250 \mu \mathrm{V} \mathrm{K}{ }^{-1}\left(\mathrm{PtCoO}_{2}\right)$ for the out-of-plane component of the thermopower at room temperature, in contrast to moderate $+5 \mu \mathrm{V} \mathrm{K}^{-1}$ in-plane [17]. Such a large anisotropy in the thermopower can give rise to

*Markus.Gruner@uni-due.de a considerable laser induced voltage, which can be exploited to design photosensors based on the transverse thermoelectric effect [18].

Both compounds are stable and have been synthesized as high quality single crystals employing the so-called methathetical reaction [15,19-22]. In $\mathrm{PtCoO}_{2}$ and $\mathrm{PdCoO}_{2}$, the Co ion is found in the trivalent $\mathrm{Co}^{3+}$ state, which shows a $S=0$ low spin configuration, while $\mathrm{Pd}$ and $\mathrm{Pt}$ are monovalent [20,23-26]. Experiment [23,26-28] and first-principles theory [16,29-31] show consistently that the Fermi level of $\mathrm{PdCoO}_{2}$ is populated by Pd states, while oxygen states are scarce and Co states are essentially absent. Photoemission spectroscopy confirms the validity of this picture also for $\mathrm{PtCoO}_{2}$ [24]. Thus the high in-plane conductivity arises from the hybridized noble metal $4 d$ and $5 s$ electrons which contribute states at the Fermi level, whereas the $\mathrm{CoO}_{6}$ octahedra can be thought to form an insulating layer, inhibiting transport along the perpendicular $z$ axis. The absence of hybridized Co states at the Fermi level results in a quasi-two-dimensional electronic arrangement. This is reflected in a Fermi surface, which shows nearly no dispersion along $k_{z}$ and thus has a quasi-two-dimensional shape $[16,17,30,32]$. In consequence, the Fermi velocities, i.e., the gradients of $k$-resolved band structure at $E_{\mathrm{F}}$, are restricted to the $x-y$ plane, while the rather flat Fermi surface along $k_{z}$ prevents a significant contribution to the out-of-plane conductivity $\sigma_{z z}$. The predicted cross section of the Fermi surface in the shape of a closed hexagon was validated by Noh et al. [32] based on angular-resolved photoemission spectroscopy (ARPES).

In the present work we consider $\mathrm{PdCoO}_{2}$ and $\mathrm{PtCoO}_{2}$ as an intrinsically layered system serving as a simple prototype of a heterostructure considered for future thermoelectric applications. We determine the dependence of the electronic structure on epitaxial in-plane strain by means of first-principles calculations. Using this as input, we obtain the transport properties in the framework of semiclassical Boltzmann transport theory in the constant relaxation time approximation. We identify a remarkable dependence of the electronic transport of $\mathrm{PtCoO}_{2}$ on epitaxial strain, which stands in clear contrast to the 
rather uniform strain response of the elastic properties. We will show that this stunning discrepancy is related to an electronic topological transition, which, in turn, is beneficial in improving the thermoelectric performance of the material. It becomes manifest in a variation of the out-plane thermopower by a factor of three and the out-of-plane conductivity by more than one order of magnitude.

After a short survey of the computational details in Sec. II, we will shortly review the similarities in the structural behavior of expitaxially strained $\mathrm{PtCoO}_{2}$ and $\mathrm{PdCoO}_{2}$ in Sec. III A. The different strain response of the electronic structure is explained in Sec. III B, while Sec. IIIC is devoted to the immediate consequences for in-plane and out-of-plane conductivity. The thermopower and a prediction for the thermoelectric figure of merit for the strained materials are presented in Sec. III D.

\section{NUMERICAL DETAILS}

The electronic structure was investigated within the framework of density functional theory (DFT) employing the Vienna $a b$ initio simulation package (VASP) $[33,34]$, which uses a plane-wave basis set for the description of the valence electrons in combination with the projector augmented wave approach (PAW) [35]. In our calculations we considered explicitly the $2 s^{2} 2 p^{4}$ electrons for $\mathrm{O}, 3 d^{8} 4 s^{1}$ for $\mathrm{Co}, 4 p^{6} 4 d^{9} 5 s^{1}$ for $\mathrm{Pd}$, and $5 d^{9} 6 s^{1}$ for $\mathrm{Pt}$, choosing a cutoff energy of $500 \mathrm{eV}$. The exchange-correlation part of the Hamiltonian was represented by the generalized gradient approximation (GGA) of Perdew, Burke, and Ernzerhof [36].

The response of the system to epitaxial strain exerted along the $a-b$ plane was calculated from the hexagonal 12-atom unit cell within the scalar-relativistic approximation. The hexagonal basis of this cell allows a straightforward manipulation of the lattice parameters according to the epitaxial constraint. For a given value of $a$, the $c$ lattice parameter and atomic positions were optimized for minimum energy modeling the epitaxial constraint. In order to obtain accurate eigenvalues, as needed for the transport calculations described below, we transformed the 12-atom unit cell with hexagonal basis to the four-atom primitive cell with rhombohedral basis using a mesh of $41 \times 41 \times 41 k$ points. We included the spin-orbit term to the Hamiltonian in our self-consistent treatment, which yields small but noticeable changes to the results. For additional corroboration and high quality Fermi surfaces, parts of the calculations were repeated with the full-potential augmented plane-wave method Wien2k [37]. Thermoelectric properties at finite temperatures were obtained in the framework of semiclassical Boltzmann transport theory in the constant relaxation time approximation under the constraint of a conserved number of carriers. This step was carried out with the BoltzTraP [38] code based on the the eigenvalues obtained from our VASP calculations. In order to test the effect of additional correlation on the electronic structure and transport properties, we applied the GGA $+U$ scheme in the rotationally invariant formulation of Dudarev et al. [39] on the Co $d$ states for selected cases, using different values of $U-J$ up to $5 \mathrm{eV}$. Further technical details can be found in the Supplemental Material [40].

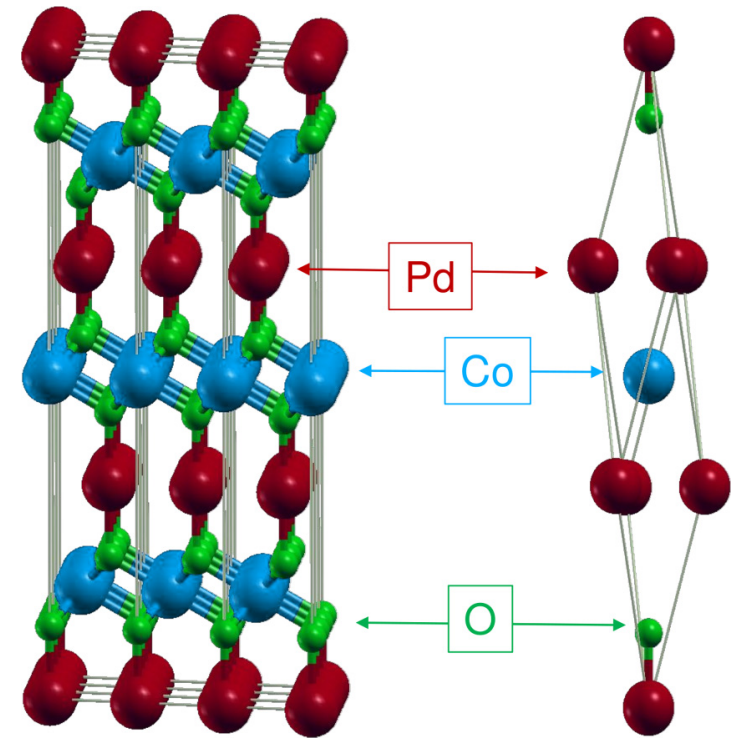

FIG. 1. (Color online) Representation of $\mathrm{PdCoO}_{2}$ and $\mathrm{PtCoO}_{2}$ delafossites in a unit cell with 12 atoms corresponding to the hexagonal basis (left image, three times replicated in $a$ and $b$ direction) and a four-atom rhombohedral primitive cell (right).

\section{RESULTS AND DISCUSSION}

\section{A. Structural properties strained $\mathrm{PdCoO}_{2}$ and $\mathrm{PtCoO}_{2}$}

The delafossites crystallize in a hexagonal structure (see Fig. 1), which can be described by the rhombohedral space group 166 with symmetry $R \overline{3} m$. Pt or Pd are found on the (1a) site at $(0,0,0)$, Co on the $(1 \mathrm{~b})$ site at $(0,0,1 / 2)$, and the two oxygen on the (2c) site at $(0,0, \pm u)$, with $u$ being the internal structural parameter. This results in a naturally layered arrangement, consisting of layers of corner-sharing $\mathrm{CoO}_{6}$ octahedra and linear O-Pd-O or, respectively, O-Pt-O dumbbells. The $\mathrm{Pd} / \mathrm{Pt}$ atoms form a hexagonal layer with the triangular faces of the $\mathrm{CoO}_{6}$ octahedra on top of the triangular facets of the $\mathrm{Pd} / \mathrm{Pt}$, such that the edge oxygen of $\mathrm{CoO}_{6}$ become part of the dumbbells.

We find the ground state of both delafossites at nearly the same lattice parameters, i.e., $a=2.870 \AA$ and $c=17.94 \AA$ for $\mathrm{PdCoO}_{2}$ and $a=2.861 \AA$ and $c=17.95 \AA$ for $\mathrm{PtCoO}_{2}$. Consequently, the atomic volume of the $\mathrm{Pd}$ compound is with $V=10.66 \AA^{3}$ /atom slightly larger than the volume of the Pt compound ( $V=10.61 \AA^{3} /$ atom). These values agree well with previous DFT investigations $[30,41]$ and experiment ( $a=2.83 \AA$ for both compounds) [20-22,42,43], with the typical overestimation of the lattice constant w.r.t. experiment by approximately $1 \%$ as a consequence of the use of the GGA for the exchange-correlation potential. Both systems show an essentially identical response to epitaxial strain. The lower panel of Fig. 2 indicates that epitaxial growth with in-plane strain along $a$ of up to $5 \%$ might be realistic, since the corresponding deformation energies are still in the range of typical thermal energies. Applying in-plane strain results in a corresponding opposite strain of the out-of-plane lattice parameter $c$, which is, however, approximately only half as large. This has the consequence that upon straining the system by $\varepsilon=\Delta a / a= \pm 4 \%$ the equilibrium volume changes 


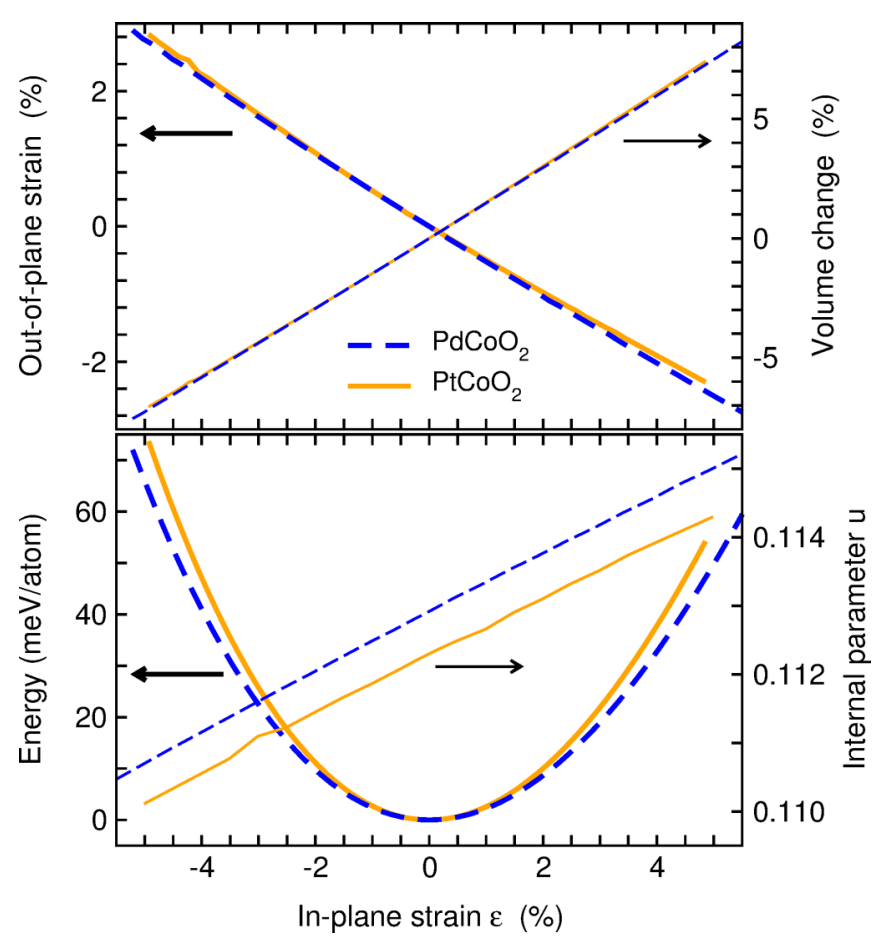

FIG. 2. (Color online) Comparison of total energy (bottom graph, left axis), internal parameter $u$ (bottom graph, right axis), out-of-plane strain (top graph, left axis) and unit cell volume (top graph, right axis) relative to the respective bulk equilibrium value as a function of the relative in-plane strain for $\mathrm{PdCoO}_{2}$ (dark blue color) and $\mathrm{PtCoO}_{2}$ (bright orange color) obtained from our first-principles calculations.

significantly by $\Delta V / V= \pm 5 \%$. The increase of $c / a$ with decreasing volume is consistent with pressure experiments on $\mathrm{PdCoO}_{2}$ [42]. Epitaxial strain also causes a variation of the internal parameter $u$, which determines the oxygen position relative to the other ions. At equilibrium conditions we find $u=1.1129$ for $\mathrm{PdCoO}_{2}$ and $u=1.1123$ for $\mathrm{PdCoO}_{2}$. This corresponds to a Pd-O distance of $2.026 \AA$ and a Pt-O distance of $2.015 \AA$ in combination with Co-O distances of 1.917 and $1.919 \AA$, respectively. $4 \%$ of tensile strain causes $u$ to increase by approximately $2 \%$. This has an opposite effect on the Co-O distance as compared to the distance between $\mathrm{O}$ and the Pt-group metal. For both oxides, the Co-O distance increases by $+1.8 \%$, while the distance between $\mathrm{O}$ and the noble metal decreases by approximately $-0.5 \%$. Under $-4 \%$ compressive strain, we observe the opposite effect with variations of $-1.5 \%$ and $+0.6 \%$, respectively.

\section{B. Electronic structure and Fermi surface of strained $\mathrm{PtCoO}_{2}$ and $\mathrm{PdCoO}_{2}$}

The band structure of the unstrained delafossites, compared in Fig. 3, suggests at first sight that the electronic features relevant for the transport properties, which appear close to the Fermi level $E_{\mathrm{F}}$, are similar for both compounds. In both cases only one band (i.e., the 16th band of valence electrons disregarding the semicore states in counting) is crossing $E_{\mathrm{F}}$ which is formed by $d_{3 z^{2}-r^{2}}$ and $s$-type orbitals with $\mathrm{Pd} / \mathrm{Pt}$ character $[16,29,30]$. Its rather steep slope in $k_{x}-k_{z}$ direction,

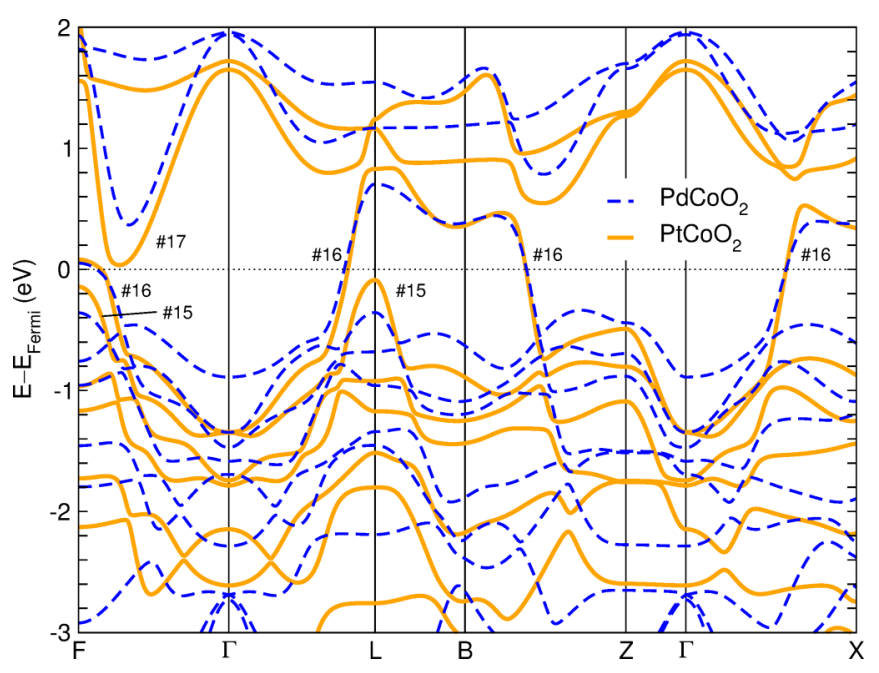

FIG. 3. (Color online) Band structure of $\mathrm{PtCoO}_{2}$ (solid orange line) and $\mathrm{PdCoO}_{2}$ (dashed blue line) in the vicinity of the Fermi level. The numbers denote the bands close to $E_{\mathrm{F}}$, which are relevant for our discussion. The counting starts from the valence band minimum (core and semicore states such as the $\mathrm{Pd} 4 p^{6}$ are not considered).

which is responsible for the excellent in-plane conductivity, corresponds to a section of a parabolic structure, which has its minimum at $\Gamma$ at $-2.6 \mathrm{eV}$ for $\mathrm{PtCoO}_{2}$ and $-2.3 \mathrm{eV}$ for $\mathrm{PdCoO}_{2}$ and is intersected by hybridizing bands with Co $d$ character several times below $E_{\mathrm{F}}$. Although most other bands of $\mathrm{PtCoO}_{2}$ are shifted downward due to the larger bandwidth related to the larger extent of the $5 d$ shell of Pt compared to the $4 d$ electrons of Pd, the 16th band does not change its shape in the immediate vicinity of $E_{\mathrm{F}}$.

This is different for the next lower, completely filled band (i.e., the 15 th band), which forms hole pockets in the vicinity of the zone boundary, in particular, near the special points $F$ and $L$. The next higher unoccupied band (17th band) displays a marked minimum (electron pocket) along $\Gamma-F$, which comprises Co $e_{g}$ states $[29,30]$ that are absent in the vicinity of $E_{\mathrm{F}}$ otherwise. In $\mathrm{PtCoO}_{2}$, these features of the 15th and the 17th band come very close to $E_{\mathrm{F}}$ within $100 \mathrm{meV}$, while in $\mathrm{PdCoO}_{2}$ they are separated from the Fermi surface by more than $300 \mathrm{meV}$. As we will show below, these fine details of the electronic structure give rise to qualitatively different transport properties under epitaxial strain which stand in contrast to the rather uniform elastic response of both systems.

The energy minimum along $\Gamma-F$ in the 17th band might be interpreted as a consequence of the hybridization between the Co $3 d_{e_{g}}$ bands with the parabolic section of the 16th band [see the Co-resolved band structure of $\mathrm{PtCoO}_{2}$ in Figs. 4(d)-4(f)], which becomes manifest in a sharp peak in the electronic density of states $0.4 \mathrm{eV}$ above the Fermi level (see Ref. [40] as well as Refs. [29,30] for the density of states and orbital resolved band plots), while the Co $3 d_{t_{2 g}}$ and the $\mathrm{Pd} 4 d_{x z}$ and $4 d_{y z}$ states are located $0.5-2 \mathrm{eV}$ below $E_{\mathrm{F}}$. In comparison, the hybridization between Pt $5 d_{3 z^{2}-r^{2}}$ and Co $3 d_{e_{p}}$ states is less pronounced and the band crossing is encountered in the immediate vicinity of the Fermi surface. A strain-induced change of the Brillouin zone can influence the position of this crossing and thus shows significant impact on the shape of 
(a)

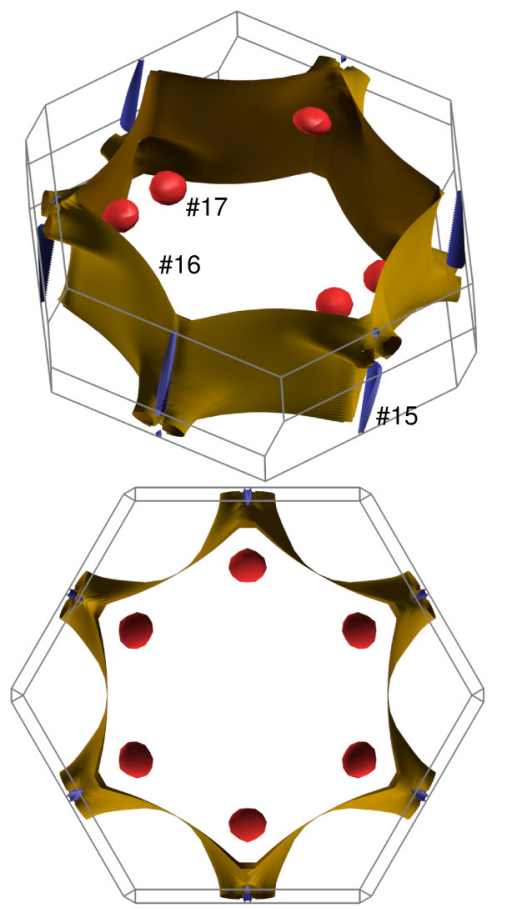

(d)

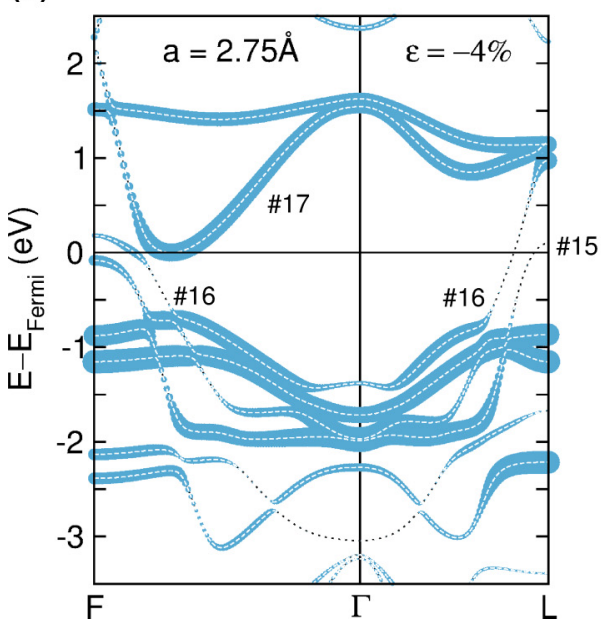

(b)

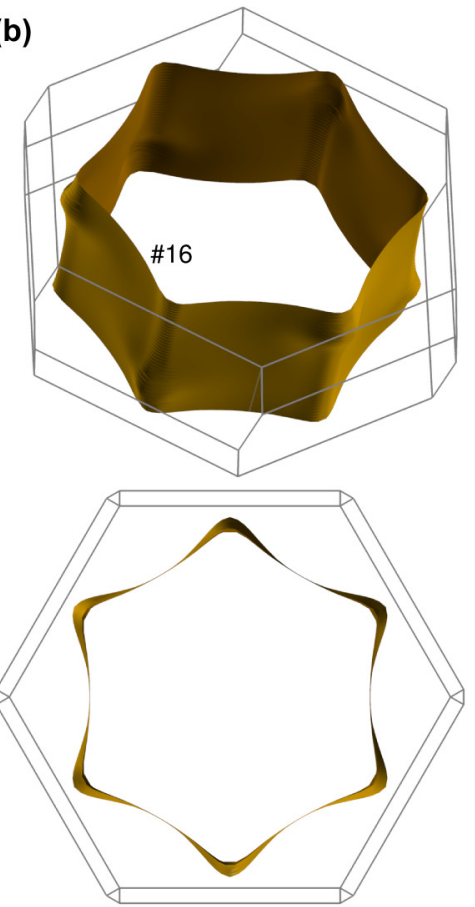

(e)

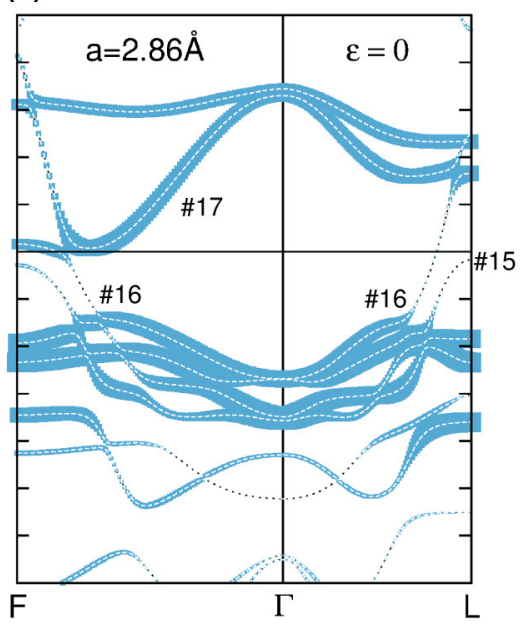

(c)
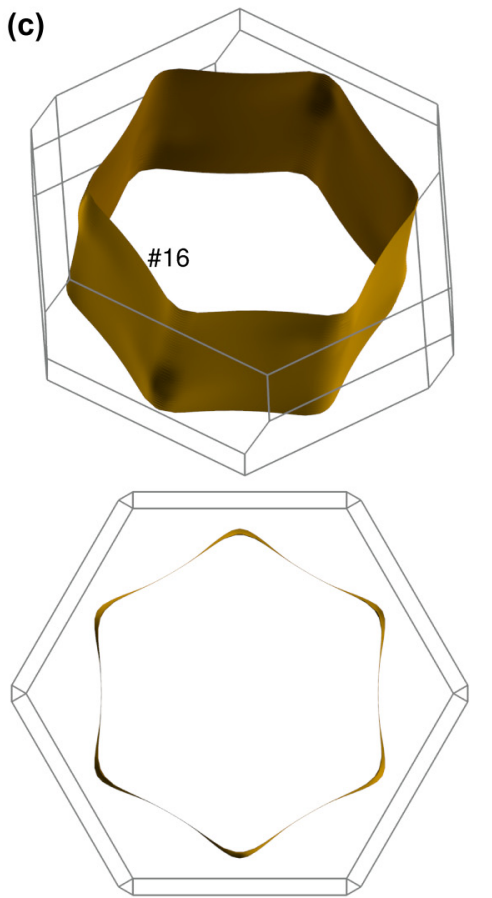

(f)

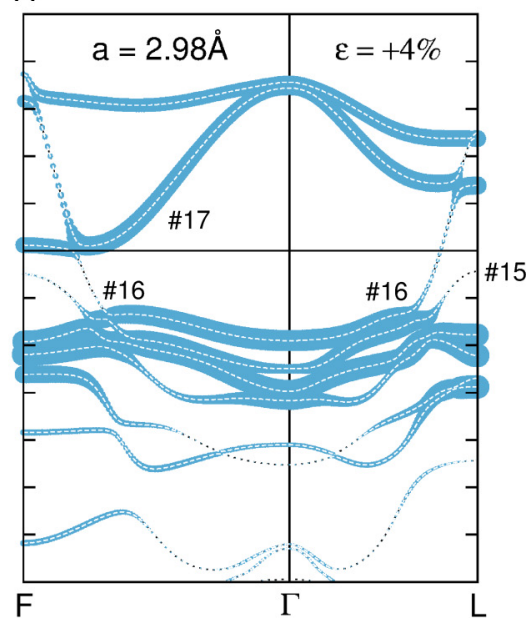

FIG. 4. (Color online) Fermi surfaces [top and center rows, (a)-(c)] and corresponding band structure [bottom row, (d)-(f)] of strained and unstrained $\mathrm{PtCoO}_{2}$. The left column [(a) and (d)] refers to $\varepsilon=-4 \%$ compressive epitaxial strain, the right column [(c) and (f)] to $\varepsilon=+4 \%$ tensile strain, and the center column [(b) and (e)] to the unstrained case. In the images of the Fermi surfaces the different colors refer to the different bands crossing the Fermi levels (blue: 15th band, orange: 16th band, red: 17th band). The vertical width of the (bright blue) fat bands in the bottom row is proportional to the Co character of the band. Again, the bands crossing $E_{\mathrm{F}}$ are denoted by their respective number. We find that the 15th and 17th bands cross the Fermi energy at compressive strains, whereas for tensile strains, the dispersion of the 16th band at $E_{\mathrm{F}}$ is influenced by the nearby crossing of a parabolic section of the 16th Pt band and the 17th band which is dominated by Co states.

the Fermi surface. This might be exploited to alter the transport properties by applying external fields, such as mechanical stress.

The crossing of the 16th band results in a hexagonal rodlike shape of the Fermi surface [Fig. 4(b)], which is a pronounced feature of both compounds [16,17,30-32]. Under a moderate compressive epitaxial strain of $\varepsilon=-4 \%$, the extremal features of the 15th and 17th bands of $\mathrm{PtCoO}_{2}$ touch the Fermi surface, leading to a significant change in topology. This is depicted in Figs. 4(a) and 4(d). The (blue) needle-type features outside the (orange) hexagonal rod evolve from the rising of the hole pocket in the 15th band, which is best seen at the $L$ point. At the same time, the 17th band approaches the Fermi level along $\Gamma-F$ from above resulting in the (red) pills inside the hexagonal rods. According to its Pt-type band character (not shown), we expect the 15th band to contribute predominantly to the in-plane conductivity. In turn, the 17th band shows a strong Co character close to its minimum where the electron pocket forms and is consequently expected to increase the out-of-plane conductivity (see Sec. IIIC). While the faces of the hexagon formed by the 16th band are essentially dispersionless irrespective of the strain, we find 
bulblike extrusions at its rounded corners. The extrusions are also present on the Fermi surface of $\mathrm{PdCoO}_{2}$ and are again associated with a slight admixture of Co states in band 16 along $\Gamma-F$ [44]. In $\mathrm{PtCoO}_{2}$ these features grow under compressive strain and eventually touch the Brillouin zone boundary, while they become significantly flatter under tensile strain, in turn. This corresponds to a diminished band velocity component in $z$ direction. Tensile epitaxial strain is thus a suitable possibility to control the $\mathrm{Pd} / \mathrm{Pt}$ character of the 16 th band and thus enhance the two-dimensional nature of the electronic structure. In reverse, compressive strain can stimulate an electronic topological transition, which arises from the appearance of additional bands at the Fermi level and the 16th band touching the Brillouin zone boundary. In $\mathrm{PdCoO}_{2}$, the distance between the $\mathrm{Co}$ and $\mathrm{Pd}$ states along $\Gamma-F$ is significantly larger, which makes the electronic states at the Fermi level much less susceptible to strain. However, electron doping may provide an alternative way to bring the systems close to this instability and produce a Fermi surface of similar shape [45].

Based on experimental evidence, $\mathrm{PtCoO}_{2}$ and $\mathrm{PdCoO}_{2}$ were considered as weakly correlated oxides [46]. Furthermore, Ong et al. $[16,17]$ found a very good agreement between the firstprinciples electronic structure and experimental spectroscopy, which justifies the use of conventional GGA for the exchange and correlation part of the Hamiltonian. However, recently, Hicks et al. argued that static Coulomb repulsion in terms of a Hubbard model with an effective $U$ parameter on the Co $3 d$ states improves the agreement with their de-Haas-van-Alphen data [47]. Therefore, we carried out additional GGA $+U$ calculations for $\mathrm{PdCoO}_{2}$ and $\mathrm{PtCoO}_{2}$ under a systematic variation of the effective Coulomb repulsion $U_{\text {eff }}=U-J$ on Co $3 d$ from 0 (pure GGA) to $5 \mathrm{eV}$. The additional term mainly affects the bonding $3 d$ Co $t_{2 g}$ states below $E_{\mathrm{F}}$ which move further down, while we observe only a minute shift in the position of the relevant Co $e_{g}$ states, which are close to $E_{\mathrm{F}}$. This separates occupied and unoccupied $\mathrm{Co}$ and $\mathrm{O}$ states further, but hardly affects the Pd states at the Fermi surface, which are responsible for metallicity. Thus $U_{\text {eff }}$ has only a minor effect on the position of the Pt-dominated 15th band but larger values will eventually inhibit the crossing of the 17 th band or shift it to larger strains.

It is worthwhile to note that the occurrence of the electronic topological transition is independent of the calculation method and likewise reproduced with VASP and Wien2k. Nevertheless, concerning the transport calculations we obtain slight differences between the codes. Interestingly, we find a closer agreement of the GGA results obtained from the Wien2k calculations with the VASP calculations for $U_{\text {eff }}=$ $1 \mathrm{eV}$, rather than for the pure GGA, although the same exchange-correlation functional (PBE) was used in both cases. We ascribe this to the specific implementation of the Co PAW-potential employed in the VASP calculations, which apparently allows for a somewhat stronger interaction between $\mathrm{Co}$ and $\mathrm{Pd}$ states resulting in a slightly increased presence of residual Co states at the Fermi level compared to the full-potential calculations. For additional details see Ref. [40].

\section{Electronic conductivity under epitaxial strain}

Starting from the electronic eigenvalues obtained with DFT, we determined the transport properties in the framework of semiclassical Boltzmann transport theory using the constant relaxation time approximation. This approach has evolved as a standard tool for the prediction and identification of qualitative trends in a wide variety of materials $[48,49]$, including the prediction of oxidic systems [50,51], and has been applied successfully to several delafossites in the past [52,53]. Concerning $\mathrm{PdCoO}_{2}$ and $\mathrm{PtCoO}_{2}$, particular emphasis was laid on the explanation of the large anisotropy in conductivity and thermopower (Seebeck coefficient) [16,17,44], but also on the description of the large out-of-plane magnetoresistance encountered in $\mathrm{PdCoO}_{2}$ under the rotation of a large in-plane magnetic field [54]. The relaxation time approximation, with a single energy- and momentum-independent relaxation time, assumes that the combined effect of all scattering processes is such that the electronic system relaxes back to equilibrium exponentially with a single time constant once the perturbation is switched off. Alternatively, one may consider this approach as a first step towards the complete description which clarifies the band structure related aspects of transport. In any case, the relaxation time approximation allows for solving the Boltzmann equation in a simple way, and hence an accurate determination of transport coefficients using $10^{4}$ to $10^{5} k$ points, which are necessary for converged results. From the comparison with experiment, on the other hand, one is then able to deduce information on the relevant scattering mechanisms.

A more detailed theoretical description could be based on Boltzmann theory, but would require a first-principles calculation of phonon dispersions as well as electron-phonon scattering matrix elements, which is beyond the scope of the present work. Alternatively, the determination of transport coefficients can be based directly on a Green's function approach and Kubo's linear response theory (see Ref. [55] for a recent discussion).

At room temperature and above, it can be expected that electron-phonon scattering is the dominant relaxation mechanism. For the nearly free electron model, the scattering operator indeed has been studied in detail [56]. For example, it is well known that for temperatures below the Debye temperature, the momentum and energy relaxation rates are very different, the latter being much shorter than the former. Above the Debye temperature, electron-phonon scattering is essentially elastic, and both rates are very similar. These results rely, in particular, strongly on the fact that the electronic density of states (DOS) is practically independent of energy around the Fermi level. Thus predictions based on an energyindependent $\tau$ are likely to fail in case sharp features exist close to $E_{\mathrm{F}}$. This was shown recently for the simple metal $\mathrm{Li}$, where the constant relaxation time approximation predicts the wrong sign of the thermopower [57]. For Li, quantitative agreement can be achieved within a variational approach to Boltzmann theory [57-60]. Since our calculated transport properties are consistent with the available experimental data for $\mathrm{PdCoO}_{2}$, as shown below, we do not expect significant new insights from an advanced treatment of the Boltzmann equation. However, our results will demonstrate clearly that-according to the anisotropic nature of the lattice structure-one must take into account at least a directional dependence of $\tau$ in addition to its variation with temperature.

In practice, all transport tensors obtained from Boltzmann transport theory turn out to be essentially diagonal; there are 


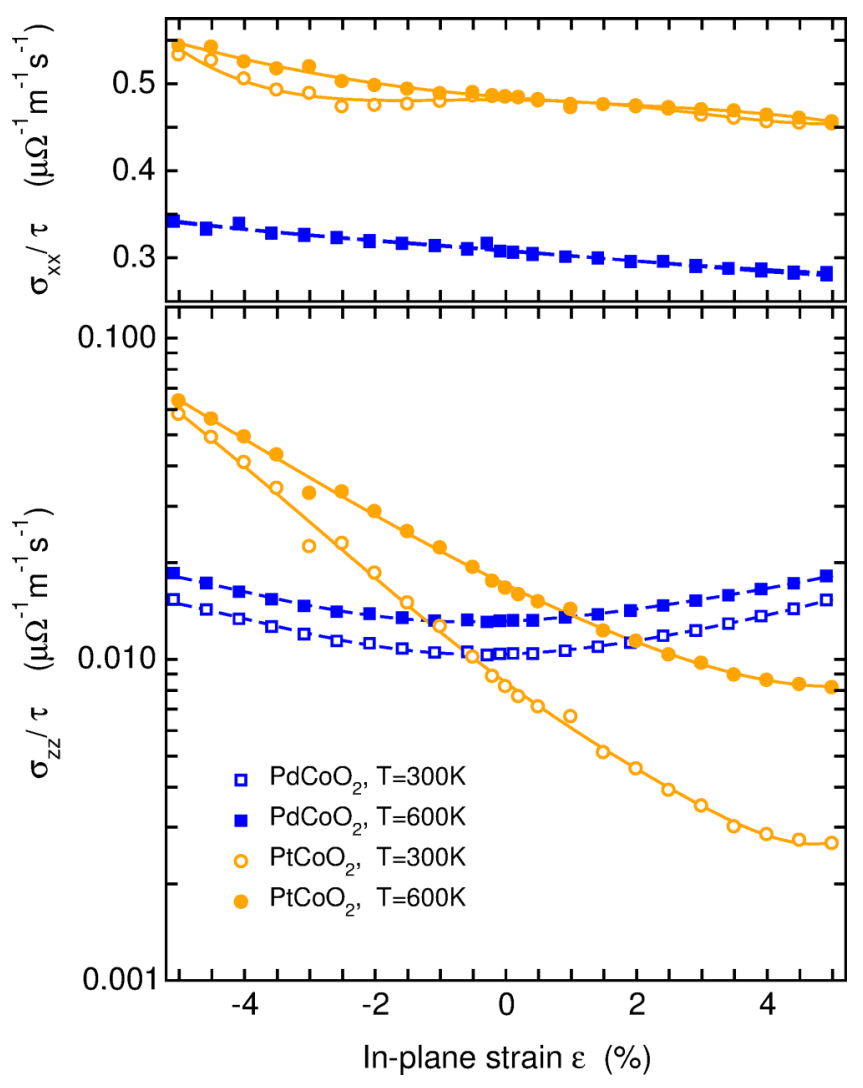

FIG. 5. (Color online) In-plane and out-of-plane components of the electrical conductivity tensor $\sigma_{x x}$ (upper panel) and $\sigma_{z z}$ (lower panel) as a function of epitaxial strain. The results are specified relative to the empirical parameter of Boltzmann transport theory, the relaxation time constant $\tau$. We compare the two different compounds $\mathrm{PdCoO}_{2}$ (dashed lines and squares) and $\mathrm{PtCoO}_{2}$ (solid lines and circles) at two different temperatures $T=300 \mathrm{~K}$ (open symbols) and $T=600 \mathrm{~K}$ (filled symbols). Please note the semilogarithmic scale in the bottom panel.

only two distinct entries. The first, marked with the index " $x x$," corresponds to in-plane transport, the second, marked as " $z z$ " corresponds to transport properties perpendicular to the $\mathrm{Co}-\mathrm{O}$ and $\mathrm{Pd}-\mathrm{O} / \mathrm{Pt}-\mathrm{O}$ layers.

As already anticipated, the significant changes of the Fermi surface of $\mathrm{PtCoO}_{2}$ should leave a corresponding signature in the strain-dependent conductivity, shown in Fig. 5. The variation of the in-plane component with strain, which accounts for the already very good conductivity in this direction, is not substantial (upper panel). The steeper slope at $E_{\mathrm{F}}$ indicates a larger ratio $\sigma_{x x} / \tau$ for $\mathrm{PtCoO}_{2}$ compared to $\mathrm{PdCoO}_{2}$. Both values are steadily reduced for tensile strains. However, as indicated earlier, the conductivity is highly anisotropic and thus orders of magnitude smaller in the perpendicular direction. Following the changes in the Fermi surface, the strain-induced variation of the out-of-plane conductivity is much more significant for bulk $\mathrm{PtCoO}_{2}$. Expanding the $a$ axis by $4 \%$ decreases the out-of-plane conductivity by almost one order of magnitude. In turn, the opposite trend is encountered for compressive strain. This significantly increases the conductivity compared to $\mathrm{PdCoO}_{2}$, where the variations are much smaller and do not exhibit a consistent trend for both strain directions.

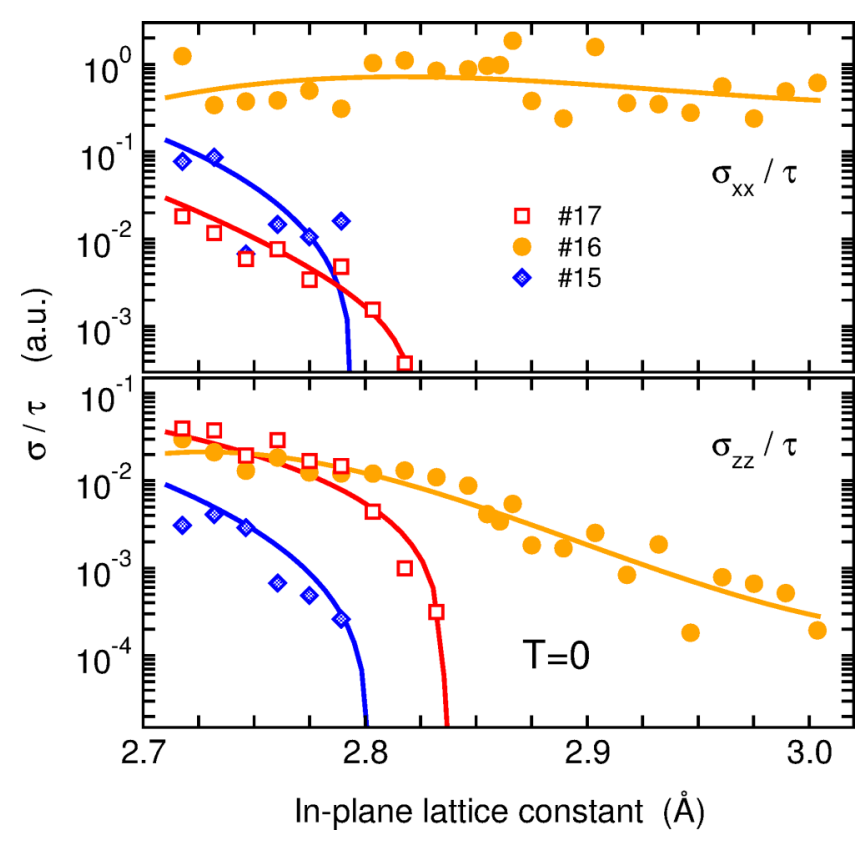

FIG. 6. (Color online) Band-resolved diagonal components $\sigma_{x x}$ and $\sigma_{z z}$ of the electronic conductivity tensor at $T=0$ of $\mathrm{PtCoO}_{2}$ as a function of the in-plane lattice constant $a$. The values are specified relative to the relaxation time constant $\tau$. Only three bands crossing $E_{\mathrm{F}}$ contribute to the transport properties.

Further insight in the strong impact of the Fermi surface on the conductivity anisotropy in $\mathrm{PtCoO}_{2}$ is obtained by the band resolved in-plane and out-of-plane components of the electrical conductivity tensor of $\mathrm{PtCoO}_{2}$ (Fig. 6). For tensile strain only the 16th band contributes since it is still the only one crossing the Fermi surface. Its in-plane component remains essentially constant, while the out-ofplane component is responsible for the exponential decrease of the conductivity shown in Fig. 5. The additional bands start contributing to the conductivity tensor below an in-plane lattice constant $a<2.80 \AA$ (15th band) and $a<2.83 \AA$ (17th band), respectively, see Fig. 6. For tensile strains, the conductivity originates from the 16th band, alone. Its strong decrease can be related to an increasingly better definition of the hexagonal corners of the Fermi surface along the $k_{z}$, as visible from the comparison of the Fermi surface under tensile strain with the equilibrium Fermi surface [Figs. 4(a)-4(c), center row]. This corresponds to a diminished band velocity component in $z$ direction, which consequently reduces the respective element of the conductivity tensor. In a simple geometric picture, this complies with the decreasing width of the conducting $\mathrm{PtO}_{2}$ layer, while the separation between $\mathrm{O}$ and $\mathrm{Co}$ and thus the width of the insulating layer becomes larger.

Experimental values for the anisotropy $\sigma_{x x} / \sigma_{z z}$ of $\mathrm{PdCoO}_{2}$ range from 150 [43], to 280 [61], and 400 [47]. This large discrepancy has been noticed earlier and related to the treatment of umklapp processes and the anisotropy of defect scattering cross sections [47]. In particular, the latter aspects point out possible shortcomings of the single relaxation time approximation and semiclassical Boltzmann transport theory. Computing the anisotropy ratio from Fig. 5 assuming a single constant $\tau$, the in-plane conductivity is a factor 30 larger than 
the out-of-plane. This ratio can be somewhat increased by applying static Coulomb correlations within the GGA $+U$ scheme (see Ref. [40] for details) but it never becomes comparable to the experimental results. We take this as another indication that a directional dependence of $\tau$, which has been neglected so far, should enter the anisotropy ratio.

The diagonal structure of the transport tensors allows us to pragmatically circumvent this problem by introducing two effective, temperature dependent relaxation time constants for in-plane and out-of-plane processes, $\tau_{x x}$ and $\tau_{z z}$, respectively, which we obtain by comparison with the experimental conductivities. This effectively re-introduces the $k$-vector dependence of $\tau$, which is discarded in Boltzmann theory within the common single relaxation time approximation. As for the anisotropy, experiment offers a considerable span of results also for the in-plane experimental conductivities $\sigma_{x x}$, ranging from $14.5 \mu \Omega^{-1} \mathrm{~m}^{-1}$ [43], over $32.3 \mu \Omega^{-1} \mathrm{~m}^{-1}$ [61] and $38.5 \mu \Omega^{-1} \mathrm{~m}^{-1}$ [47] to $50 \mu \Omega^{-1} \mathrm{~m}^{-1}$ [12]. This results in relaxation time constants $\tau_{x x}(300 \mathrm{~K})$ of $46,101,121$, and 157 fs, respectively. These comparatively large values are consistent with the previous assessment of Ong et al. [17], obtained in a similar fashion by comparing Boltzmann theory and experimental conductivity data. From the residual in-plane resistivities for $T \rightarrow 0$, Hicks et al. [47] concluded on an extremely large transport mean-free path $l_{\text {MFP }}=20 \mu \mathrm{m}$. This is considerably larger than the earlier estimate $l_{\mathrm{MFP}}=60 \AA$ of Noh et al. [32] based on the peak width obtained from ARPES. These measurements also yield a value for the in-plane carrier velocity of $4.96 \mathrm{eV} \AA \hbar^{-1}$ which is consistent with first-principles results [17]. Thus, relaxation time constants obtained from experiment were either significantly smaller, such as the 7.6 fs estimated by Noh et al. [32] or larger, based on the de-Haas-van-Alphen measurements of Hicks et al. [47] or the analysis of anomalous magnetoresistance [54]. For the out-of-plane direction, ambient $\sigma_{z z}$ was measured as 0.096 [47], 0.097 [43], and $0.115 \mu \Omega^{-1} \mathrm{~m}^{-1}$ [61], corresponding to $\tau_{z z}(300 \mathrm{~K})=9$ and $11 \mathrm{fs}$, respectively, which yields a more uniform picture than the in-plane case.

Most experiments were carried out at room temperature and below, only Takatsu et al. performed conductivity measurements up to $500 \mathrm{~K}$. Thus, only Ref. [43] offers a reasonable possibility to extrapolate the experimental conductivities to $T=600 \mathrm{~K}$. We find then $\sigma_{x x}=5 \mu \Omega^{-1} \mathrm{~m}^{-1}$ and $\sigma_{z z}=$ $0.04 \mu \Omega^{-1} \mathrm{~m}^{-1}$, which corresponds to $\tau_{x x}(600 \mathrm{~K})=16 \mathrm{fs}$ and $\tau_{z z}(600 \mathrm{~K})=4$ fs. For $\mathrm{PtCoO}_{2}$, Rogers et al. [12] reported conductivities $\sigma_{z z}=33 \mu \Omega^{-1} \mathrm{~m}^{-1}$ and $\sigma_{z z}=0.1 \mu \Omega^{-1} \mathrm{~m}^{-1}$. This leads to $\tau_{x x}(300 \mathrm{~K})=70 \mathrm{fs}$ and $\tau_{z z}(300 \mathrm{~K})=12 \mathrm{fs}$, which is fairly close to the relaxation times obtained for $\mathrm{PdCoO}_{2}$.

\section{Thermoelectric performance}

The thermopower of $\mathrm{PdCoO}_{2}$ was first reported by Yagi et al. [62] for temperatures above $500 \mathrm{~K}$. Since the authors measured on polycrystalline materials, the highly anisotropic behavior of this quantity was overseen. Later, Hasegawa et al. [63] measured the thermopower at lower temperatures, again on powdered and sintered crystals. The authors obtained a positive value of $S=2-4 \mu \mathrm{V} \mathrm{K}^{-1}$ at room temperature, which is comparable to conventional metals. In a very recent

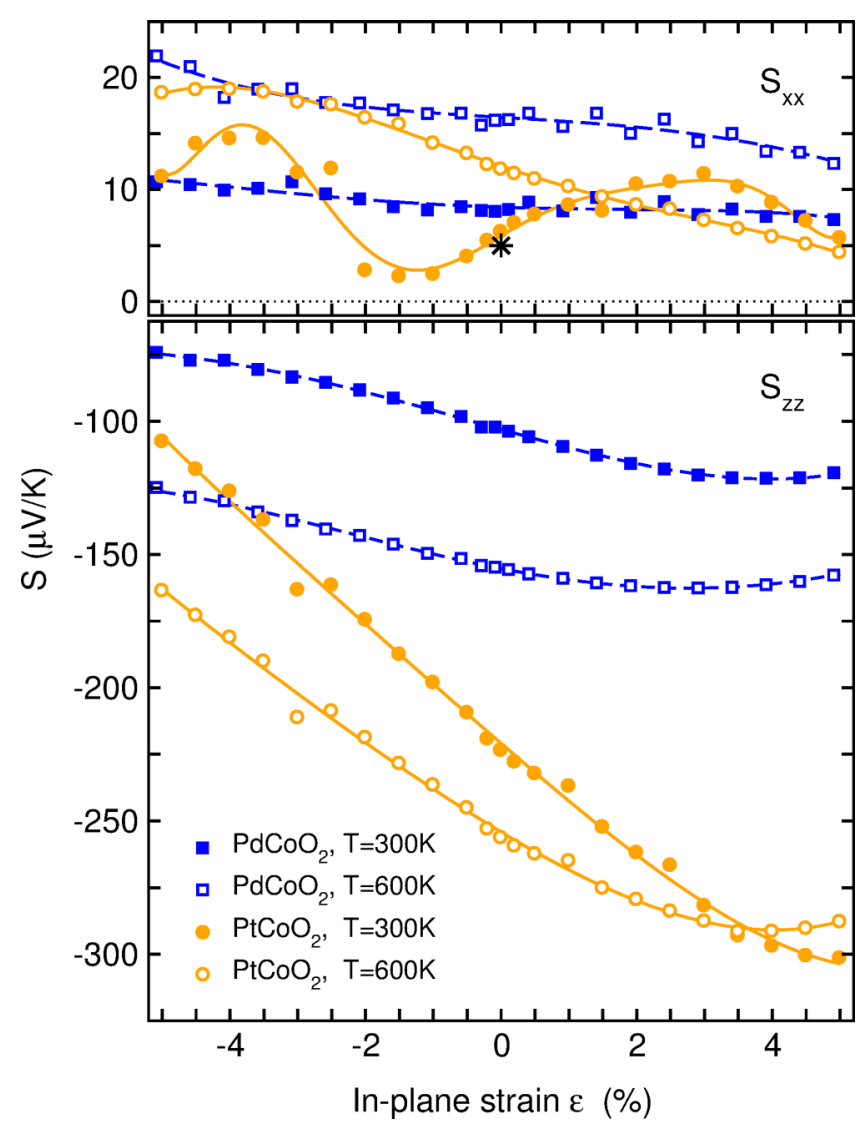

FIG. 7. (Color online) Diagonal elements $S_{x x}$ (upper panel) and $S_{z z}$ (lower panel) of the tensorial thermopower of $\mathrm{PdCoO}_{2}$ (blue dashed lines and squares) and $\mathrm{PtCoO}_{2}$ (orange solid lines and circles) as a function of the epitaxial in-plane strain for two different temperatures $T=300 \mathrm{~K}$ (open symbols) and $T=600 \mathrm{~K}$ (filled symbols). The black star in the upper panel marks the experimental in-plane thermopower of $\mathrm{PdCoO}_{2}$ at $T=300 \mathrm{~K}$ taken from Ref. [61].

study the in-plane thermopower $S_{x x}$ and thermal conductivity of $\mathrm{PdCoO}_{2}$ was measured for temperatures below $300 \mathrm{~K}$ [61]. $S_{x x}$ exhibits a change of sign below $T<100 \mathrm{~K}$, but approaches the expected linear temperature dependence at higher temperatures, reaching $S_{x x}=5 \mu \mathrm{V} \mathrm{K}^{-1}$ at ambient conditions.

Figure 7 shows the calculated thermopower (Seebeck coefficient) $S$ as a function of the epitaxial strain for both directions and two different temperatures $T=300$ and $600 \mathrm{~K}$. For both systems we confirm the marked anisotropy in the Seebeck coefficient, which is significantly more pronounced for $\mathrm{PtCoO}_{2}$ than for $\mathrm{PdCoO}_{2}$ and increases with temperature. It is positive for the in-plane component $S_{x x}$, which indicates a higher mobility of $p$-type carriers (holes), while large negative values for $S_{z z}$ point our the dominance of $n$-type carriers (electrons) for transport in out-of-plane direction. The estimate for $S_{x x}$ of unstrained $\mathrm{PdCoO}_{2}$ from Boltzmann theory at room temperature is approximately 1.6 times larger than the experimental value published in Ref. [61]. This might still be considered a reasonable agreement keeping in mind the simplifications of Boltzmann transport theory and experimental difficulties in obtaining this quantity. Previous calculations by Ong et al. [16,17] report moderate positive values in-plane for $S_{x x}$, while the out-of-plane component $S_{z z}$ 
provides a large negative contribution. Our values compare well to the results of Ong et al. [17], who carried out their calculations with the Wien $2 \mathrm{k}$ code for the experimental lattice constant, which differs slightly from our setup.

We now turn to the effect of strain, which is varied from $-5 \%$ compressive to $+5 \%$ tensile strain. The in-plane thermopower $S_{x x}$ and its variation under strain remains moderate in absolute numbers. We achieve changes in the range of $10-12 \mu \mathrm{V} / \mathrm{K}$ at $T=600 \mathrm{~K}$ as compared to $\varepsilon=0$ for both systems. Nevertheless, as the absolute values are small, maximum strain corresponds to a relative change by a factor of 2 for the Pt-based oxide. The oscillations observed at lower temperatures are a consequence of the electronic topological transition related to the two additional bands consecutively crossing the Fermi level. For the out-of-plane component $S_{z z}$, we find much larger changes in absolute numbers, in particular, around room temperature. Here the tensile strain yields a relative increase of $60 \%$ to $180 \%$, which corresponds to rather significant changes in absolute numbers of -47 and $-196 \mu \mathrm{V} / \mathrm{K}$ for $\mathrm{PdCoO}_{2}$ than for $\mathrm{PtCoO}_{2}$, respectively.

In particular, for $\mathrm{PtCoO}_{2}$, the variation of the out-of-plane thermopower $S_{z z}$ with strain bears close similarities with the logarithm of the strain dependence of the conductivity element $\sigma_{z z}$. Such a relation is motivated by the textbook formula of Mott, which connects the scalar thermopower $S$ of an isotropic system with the logarithmic derivative of the (scalar) conductivity $\sigma$ with respect to the chemical potential $\mu$, where $e$ is the electron charge:

$$
S=\frac{\pi^{2} k_{\mathrm{B}}^{2} T}{3 e} \frac{\partial}{\partial \mu} \ln [\sigma(\mu)] .
$$

We therefore conclude that the large strain variations in the thermopower originate from the corresponding changes in the conductivity discussed above. The negative sign of $S_{z z}$ is related to the decrease of $\sigma_{z z}$ with increasing chemical potential $\mu$ at a given strain [44], while the strong variation of the magnitude of $\left|S_{z z}\right|$ with strain relates inversely to the change in conductivity.

The figure of merit of a material with respect to its thermoelectric performance is given by the dimensionless number $Z T=S^{2} \sigma T /\left(\kappa^{\mathrm{el}}+\kappa^{\mathrm{ph}}\right)$, where $\kappa^{\mathrm{el}}$ and $\kappa^{\mathrm{ph}}$ are the electronic and lattice thermal conductivity, respectively. Apart from $\kappa^{\mathrm{ph}}$, all quantities are accessible within our approach. However, $\kappa^{\text {ph }}$ becomes the dominant contribution when the electrical conductivity is minute, e.g., in semiconductors or insulators, and will thus be relevant for the out-of-plane transport. The calculation of $\kappa^{\mathrm{ph}}$ from first-principles requires the determination of the anharmonic contributions to lattice dynamics, which is beyond the scope of the present work. However, the total and lattice contributions to the thermal conductivity of $\mathrm{PdCoO}_{2}$ were recently obtained experimentally by Daou et al. [61] for temperatures up to $320 \mathrm{~K}$. For the in-plane case, the authors reported a rather large total thermal conductivity $\kappa_{x x}^{\text {tot }}$ of $250 \mathrm{~W} \mathrm{~K}^{-1} \mathrm{~m}^{-1}$, while the total out-of-plane conductivity $\kappa_{z z}^{\text {tot }}$ amounts to approximately $70 \mathrm{~W} \mathrm{~K}^{-1} \mathrm{~m}^{-1}$ at ambient conditions. According to the Wiedemann-Franz law, the electronic contribution $\kappa_{z z}^{\mathrm{el}}$ is expected to be at least one order of magnitude smaller, based on the very low out-of-plane

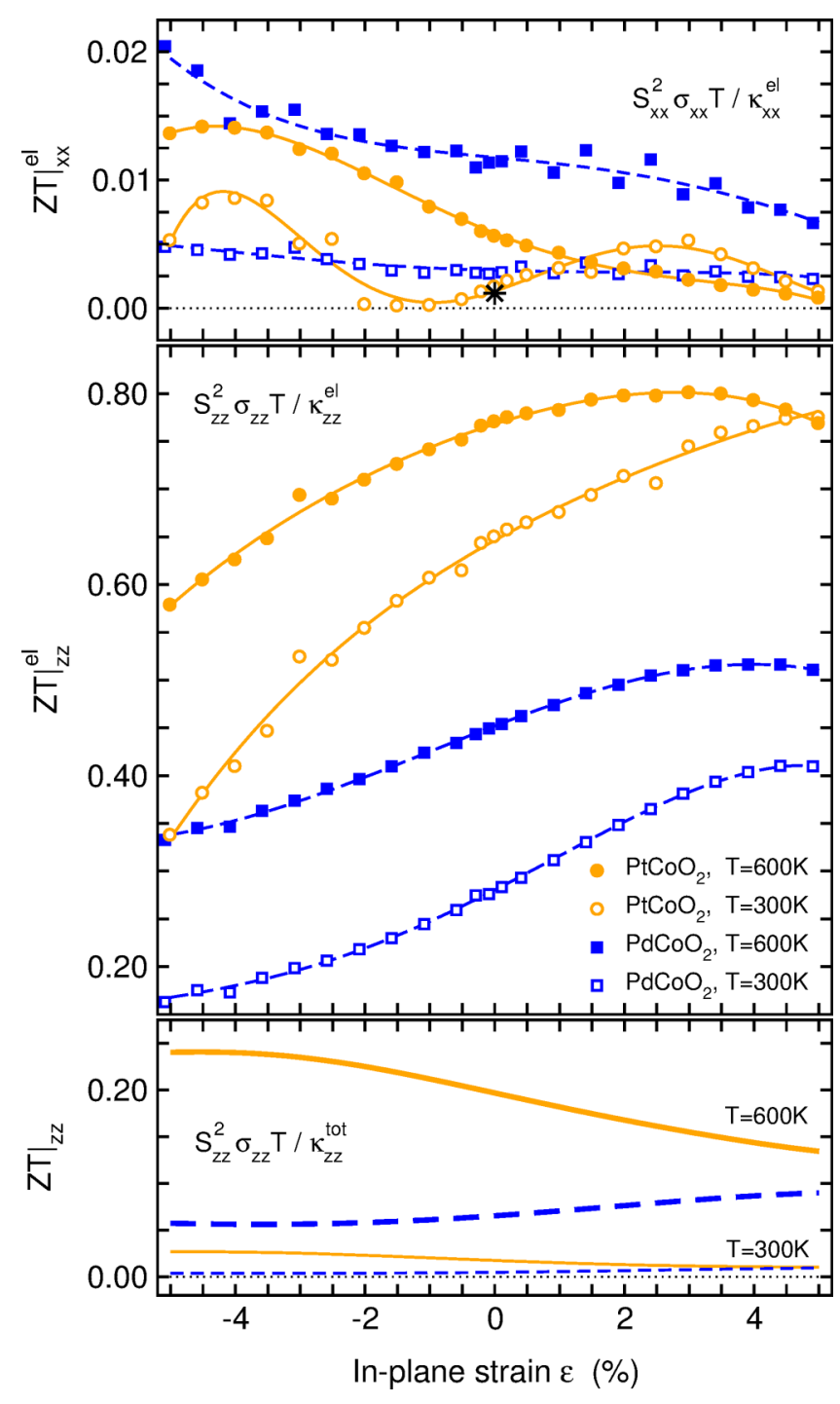

FIG. 8. (Color online) Thermoelectric figure of merit $Z T$ calculated from the diagonal elements of the transport tensors (upper panel, $x x$ elements; lower panel $z z$ elements, symbols and colors as in the preceding figures). Thin lines and data points in the upper and middle panel denote an upper boundary $\left.Z T\right|^{\mathrm{el}}$ as lattice thermal conductivity is neglected. The bottom panel shows an estimate of the total $Z T$ using the experimental $\kappa_{z z}^{\mathrm{ph}}$ for $\mathrm{PdCoO}_{2}$ from Ref. [61] (see text) and the relaxation time constant $\tau=10$ fs (same values for both compounds and all strains). The thin lines refer to $T=300 \mathrm{~K}$ and the thick lines to $T=600 \mathrm{~K}$, while broken lines denote $\mathrm{PdCoO}_{2}$ and solid lines $\mathrm{PtCoO}_{2}$. The black star in the upper panel marks the experimental in-plane $Z T$ of $\mathrm{PdCoO}_{2}$ at $T=300 \mathrm{~K}$ calculated from the experimental data of Daou and co-workers [61].

conductivity. This yields thus a direct estimate of the lattice thermal conductivity $\kappa_{z z}^{\mathrm{ph}}$.

According to the Wiedemann-Franz law, the electronic conductivities $\kappa^{\mathrm{el}}$ increase with temperature, whereas for the lattice thermal conductivity $\kappa^{\mathrm{ph}}$ a fast decrease is expected according to the Debye-Callaway model. For the in-plane component of $Z T$, we can neglect $\kappa^{\mathrm{ph}}$ as a first approximation since it is significantly exceeded by $\kappa^{\mathrm{el}}$. The corresponding quantity, which we denote by $\left.Z T\right|^{\mathrm{el}}$, provides thus an upper 
limit for the true $Z T$. According to the large thermal conductivity, the in-plane values turn out to be prohibitively low from the application point of view. Since the strain dependence of $\sigma$ and $\kappa^{\mathrm{el}}$ essentially cancels out, the strain dependence of $\left.Z T\right|_{x x} ^{\mathrm{el}}$ is dominated by the contribution from $S_{x x}^{2}$ (cf. Fig. 8). This is also the case for $\left.Z T\right|_{z z} ^{\mathrm{el}}$. However, since the thermal conductivity is dominated by the lattice contribution, we cannot use $\left.Z T\right|_{z z} ^{\mathrm{el}}$ to assess the thermoelectric performance of the material. Instead, we determine the complete $\left.Z T\right|_{z z}$ with the help of the experimental value $\kappa_{z z}^{\mathrm{ph}}$ of Ref. [61] for $T=300 \mathrm{~K}$ and $\kappa_{z z}^{\mathrm{ph}}=10 \mathrm{~W} \mathrm{~K}^{-1} \mathrm{~m}^{-1}$ for $T=600 \mathrm{~K}$, which can be estimated from the fit to the Debye-Callaway model provided in Ref. [61]. In addition, we use an average $\tau_{z z}=10 \mathrm{fs}$ for $T=300 \mathrm{~K}$, while for $T=600 \mathrm{~K}$ we take $\tau_{z z}=4 \mathrm{fs}$, as discussed in Sec. III C. As there is only sufficient data for $\mathrm{PdCoO}_{2}$, we use the same parameters for both systems. The strain dependence of $\left.Z T\right|_{z z}$ is plotted as thick dashed $\left(\mathrm{PdCoO}_{2}\right)$ and solid $\left(\mathrm{PtCoO}_{2}\right)$ lines in the bottom part of Fig. 8 . Since the dominant contribution to thermal conductivity comes from the lattice, which we assume constant for all $\varepsilon$, the strain dependence of $\left.Z T\right|_{z z}$ rather resembles that of the power factor (see Ref. [40]). As a general trend, we see that $\left.Z T\right|_{z z}$ increases strongly with temperature, while $\mathrm{PtCoO}_{2}$ clearly exceeds the performance of $\mathrm{PdCoO}_{2}$. Thus, only $\mathrm{PtCoO}_{2}$ can reach a reasonable figure of merit of $\left.Z T\right|_{z z}=0.25$ at compressive epitaxial strains and sufficiently high temperatures, which is a consequence of the electronic topological transition.

\section{CONCLUSIONS}

Based on first-principles calculations in combination with Boltzmann transport theory in the single relaxation time approximation, we provide a systematic analysis of the electronic structure and anisotropic transport properties of the delafossites $\mathrm{PdCoO}_{2}$ and $\mathrm{PtCoO}_{2}$ under epitaxial strain. We demonstrate that despite the large similarities in both systems concerning their structural properties that $\mathrm{PtCoO}_{2}$ has - unlike $\mathrm{PdCoO}_{2}$ - the propensity to undergo an electronic topological transition, which might be triggered by a realistic compressive epitaxial strain. In turn, by expanding the in-plane lattice constant, it exhibits a dimensional crossover from a three-dimensional open Fermi surface, which touches the zone boundary in-plane and out-of-plane, to a nearly perfect two-dimensional electronic system, with a closed hexagonal Fermi surface with perfectly flat sides extending in $k_{z}$ direction.
Comparing the in-plane and out-of-plane conductivities from our calculations based on GGA correlation and the $\mathrm{GGA}+U$ approach with experiment, we propose that two different relaxation times must be introduced to describe inplane and out-of-plane transport appropriately in Boltzmann transport theory. We consider this approach justified in the present case due to the essentially diagonal structure of the transport tensors.

We finally predict that, despite the apparent similarities of both oxides, $\mathrm{PtCoO}_{2}$ exhibits a much better thermoelectric performance than $\mathrm{PdCoO}_{2}$ and might thus be a better model system for applications. Our analysis includes the thermoelectric figure of merit $Z T$, which we obtain by combining theoretical results with recently available experimental data for the lattice thermal conductivity of $\mathrm{PdCoO}_{2}$ [61]. The presence of the topological transition significantly improves $Z T$, since it increases the out-of-plane conductivity, which can be tuned efficiently by an external control parameter, such as epitaxial strain. In this way we can balance the contributions of electronic and lattice thermal conductivity effectively, while still allowing for sufficiently large absolute values of the thermopower. With this strategy we arrive at a reasonable figure of merit of $Z T=0.25$ at $T=600 \mathrm{~K}$ in out-of-plane direction for a system under compressive in-plane strain. For tensile strains the out-of-plane conductivity becomes very low and $Z T$ small, since the lattice thermal conductivity dominates. But since the thermopower is almost three times larger than for compressive strains, appropriate doping of carriers may still improve the thermoelectric performance reasonably.

In conclusion, the results for $\mathrm{PtCoO}_{2}$ as a model system confirm that metallic materials characterized by a quasitwo-dimensional Fermi surface may be well suited for thermoelectric applications. However, we emphasize that for a reasonable performance the two-dimensional shape must not be too perfect. In the present case, epitaxial strain was used effectively as an external parameter to obtain a certain level of imperfection that optimizes the relation of electrical and thermal conductivities in the figure of merit.

\section{ACKNOWLEDGMENTS}

The authors gratefully acknowledge useful discussions with H.-U. Habermeier, B. Keimer (Stuttgart), and M. Verstraete (Liège). Financial support was granted by the Deutsche Forschungsgemeinschaft in the framework of TRR 80 (project G8).
[1] C. Wood, Rep. Prog. Phys. 51, 459 (1988).

[2] G. J. Snyder and E. S. Toberer, Nat. Mater. 7, 105 (2008).

[3] L. D. Hicks and M. S. Dresselhaus, Phys. Rev. B 47, 12727 (1993).

[4] R. Venkatasubramanian, E. Siivola, T. Colpitts, and B. O'Quinn, Nature (London) 413, 597 (2001).

[5] P. F. P. Poudeu, J. D'Angelo, A. D. Downey, J. L. Short, T. P. Hogan, and M. G. Kanatzidis, Angew. Chem. Int. Ed. Engl. 45, 3835 (2006).
[6] H. Ohta, S. Kim, Y. Mune, T. Mizoguchi, K. Nomura, S. Ohta, T. Nomura, Y. Nakanishi, Y. Ikuhara, M. Hirano et al., Nat. Mater. 6, 129 (2007).

[7] I. Terasaki, Y. Sasago, and K. Uchinokura, Phys. Rev. B 56 , R12685 (1997).

[8] K. Fujita, T. Mochida, and K. Nakamura, Jpn. J. Appl. Phys. 40, 4644 (2001).

[9] M. Shikano and R. Funahashi, Appl. Phys. Lett. 82, 1851 (2003). 
[10] K. Koumoto, I. Terasaki, and R. Funahashi, MRS Bull. 31, 206 (2006).

[11] J. He, Y. Liu, and R. Funahashi, J. Mater. Res. 26, 1762 (2011).

[12] D. B. Rogers, R. D. Shannon, C. T. Prewitt, and J. L. Gillson, Inorg. Chem. 10, 723 (1971).

[13] R. Nagarajan, N. Duan, M. K. Jayaraj, J. Li, K. A. Vanaja, A. Yokochi, A. Draeseke, J. Tate, and A. W. Sleight, Int. J. Inorg. Mater. 3, 265 (2001).

[14] M. A. Marquardt, N. A. Ashmore, and D. P. Cann, Thin Solid Films 496, 146 (2006).

[15] M. Tanaka, M. Hasegawa, and H. Takei, J. Phys. Soc. Jpn. 65, 3973 (1996)

[16] K. P. Ong, J. Zhang, J. S. Tse, and P. Wu, Phys. Rev. B 81, 115120 (2010).

[17] K. P. Ong, D. J. Singh, and P. Wu, Phys. Rev. Lett. 104, 176601 (2010).

[18] G. W. Yan, L. Yu, Y. Wang, H. Zhang, P. X. Zhang, and H.-U. Habermeier, J. Appl. Phys. 110, 103102 (2011).

[19] R. D. Shannon and C. T. Prewitt, J. Inorg. Nucl. Chem. 32, 1427 (1970).

[20] R. D Shannon, D. B. Rogers, and C. T. Prewitt, Inorg. Chem. 10, 713 (1971).

[21] C. Prewitt, R. Shannon, and D. Rogers, Inorg. Chem. 10, 719 (1971).

[22] M. Tanaka, M. Hasegawa, and H. Takei, J. Crystal Growth 173, 440 (1997).

[23] M. Tanaka, M. Hasegawa, T. Higuchi, T. Tsukamoto, Y. Tezuka, S. Shin, and H. Takei, Physica B 245, 157 (1998).

[24] T. Higuchi, D. Baba, Y. Yokoyama, M. Hasegawa, M. Tanaka, H. Takei, and A. Fukushima, Jpn. J. Appl. Phys. 42, 5698 (2003).

[25] T. Higuchi, M. Hasegawa, M. Tanaka, H. Takei, S. Shin, and T. Tsukamoto, Jpn. J. Appl. Phys. 43, 699 (2004).

[26] H.-J. Noh, J. Jeong, J. Jeong, H. Sung, K. J. Park, J.-Y. Kim, H.-D. Kim, S. B. Kim, K. Kim, and B. I. Min, Phys. Rev. B 80, 073104 (2009).

[27] T. Higuchi, T. Tsukamoto, M. Tanaka, H. Ishii, K. Kanai, Y. Tezuka, S. Shin, and H. Takei, J. Electron Spectrosc. 92, 71 (1998).

[28] M. Hasegawa, T. Higuchi, M. Tanaka, T. Tsukamoto, S. Shin, and H. Takei, Mater. Trans. 42, 961 (2001).

[29] R. Seshadri, C. Felser, K. Thieme, and W. Tremel, Chem. Mater. 10, 2189 (1998).

[30] V. Eyert, R. Fresard, and A. Maignan, Chem. Mater. 20, 2370 (2008).

[31] K. Kim, H. C. Choi, and B. I. Min, Phys. Rev. B 80, 035116 (2009).

[32] H.-J. Noh, J. Jeong, J. Jeong, E.-J. Cho, S. B. Kim, K. Kim, B. I. Min, and H.-D. Kim, Phys. Rev. Lett. 102, 256404 (2009).

[33] G. Kresse and J. Furthmüller, Phys. Rev. B 54, 11169 (1996).

[34] G. Kresse and D. Joubert, Phys. Rev. B 59, 1758 (1999).

[35] P. E. Blöchl, Phys. Rev. B 50, 17953 (1994).

[36] J. P. Perdew, K. Burke, and M. Ernzerhof, Phys. Rev. Lett. 77, 3865 (1996).
[37] P. Blaha, K. Schwarz, G. K. H. Madsen, D. Kvasnicka, and J. Luitz, WIEN2k, An Augmented Plane Wave + Local Orbitals Program for Calculating Crystal Properties (Karlheinz Schwarz, Techn. Universität Wien, Austria, 2001).

[38] G. K. H. Madsen and D. J. Singh, Comput. Phys. Commun. 175, 67 (2006)

[39] S. L. Dudarev, G. A. Botton, S. Y. Savrasov, C. J. Humphreys, and A. P. Sutton, Phys. Rev. B 57, 1505 (1998).

[40] See Supplemental Material at http://link.aps.org/supplemental/ 10.1103/PhysRevB.92.235140 for technical details, additional transport properties and numerical results of our GGA $+U$ calculations.

[41] S. Kumar, H. C. Gupta, and Karandeep, J. Phys. Chem. Solids 74, 305 (2013).

[42] M. Hasegawa, M. Tanaka, T. Yagi, H. Takei, and A. Inoue, Solid State Commun. 128, 303 (2003).

[43] H. Takatsu, S. Yonezawa, S. Muori, S. Nakatsui, K. Tanaka, and Y. Maeno, J. Phys. Soc. Jpn. 76, 104701 (2007).

[44] K. Kim, J. Kim, and B. Il Min, J. Phys. Soc. Jpn 83, 124708 (2014).

[45] C. Felser, K. Thieme, and R. Seshadri, J. Mater. Chem. 9, 451 (1999).

[46] M. Itoh, M. Mori, M. Tanaka, and H. Takei, Physica B 259-261, 999 (1999).

[47] C. W. Hicks, A. S. Gibbs, A. P. Mackenzie, H. Takatsu, Y. Maeno, and E. A. Yelland, Phys. Rev. Lett. 109, 116401 (2012).

[48] T. J. Scheidemantel, C. Ambrosch-Draxl, T. Thonhauser, J. V. Badding, and J. O. Sofo, Phys. Rev. B 68, 125210 (2003).

[49] L. Bjerg, G. K. H. Madsen, and B. B. Iversen, Chem. Mater. 23, 3907 (2011).

[50] P. Delugas, A. Filippetti, M. J. Verstraete, I. Pallecchi, D. Marré, and V. Fiorentini, Phys. Rev. B 88, 045310 (2013).

[51] G. B. Wilson-Short, D. J. Singh, M. Fornari, and M. Suewattana, Phys. Rev. B 75, 035121 (2007).

[52] D. J. Singh, Phys. Rev. B 76, 085110 (2007).

[53] A. Maignan, V. Eyert, C. Martin, S. Kremer, R. Frésard, and D. Pelloquin, Phys. Rev. B 80, 115103 (2009).

[54] H. Takatsu, J. J. Ishikawa, S. Yonezawa, H. Yoshino, T. Shishidou, T. Oguchi, K. Murata, and Y. Maeno, Phys. Rev. Lett. 111, 056601 (2013).

[55] H. Ebert, D. Ködderitzsch, and J. Minaár, Rep. Prog. Phys. 74, 096501 (2011).

[56] J. M. Ziman, Electrons and Phonons (Oxford University Press, Oxford, 1960).

[57] B. Xu and M. J. Verstraete, Phys. Rev. Lett. 112, 196603 (2014).

[58] P. B. Allen, Phys. Rev. B 17, 3725 (1978).

[59] S. Y. Savrasov and D. Y. Savrasov, Phys. Rev. B 54, 16487 (1996).

[60] B. Xu and M. J. Verstraete, Phys. Rev. B 87, 134302 (2013).

[61] R. Daou, R. Frésard, S. Hébert, and A. Maignan, Phys. Rev. B 91, 041113 (2015).

[62] H. Yagi, W.-S. Seo, and K. Koumoto, Key Eng. Mater. 181-182, 63 (2000).

[63] M. Hasegawa, I. Inagawa, M. Tanaka, I. Shirotani, and H. Takei, Solid State Commun. 121, 203 (2002). 